\title{
Consider the microbiome in the equation! They were here before us...and hosted us!
}

\author{
José-Manuel Fernández-Real ${ }^{1} \cdot$ Massimo Federici $^{2} \cdot$ Rémy Burcelin $^{3}$ \\ Published online: 21 December 2019 \\ (C) Springer Science+Business Media, LLC, part of Springer Nature 2019
}

In the 90's, inflammation captured the attention of researchers dedicated to the study of prevalent metabolic diseases [1]. Inflammation was present in patients at risk of cardiovascular disease, tightly linked to insulin resistance [2] and dyslipidemia [3], and in patients with established atherosclerosis [4]. In the search of triggers for this persistent, low-grade chronic inflammation, lipopolysaccharide (LPS) was identified as a powerful inductor of inflammation and detectable in substantial amounts in the circulation (i.e. named metabolic inflammation) of patients with metabolic disorders [5]. As LPS is present in the wall of gram negative bacteria, it was hypothesised that a specific microbiota composition could be behind the activation of the inflammatory cascade, leading in the long term to the development of metabolic diseases. In fact, penta-acylated LPS are consided anti- or with low inflammatory potential, while hexa-acylated LPS are considered as proinflammatory. Therefore, the specificity of the change in gram negative bacteria ecology could be at the origin of metabolic inflammation.

Today, there is increasing evidence that the microbiota should be considered in the pathophysiology of multiple metabolic diseases in tight interaction with diet. In this issue of REMD, ketogenic diets [6] and dietary lipids [7] are envisioned as important modulators of gut microbiota composition and a myriad of downstream factors.

It has been recently recognized that some bacteria may modulate inflammatory activity bi-directionally through the production of metabolic compounds (succinate) [8]. In addition to the release of soluble molecules such as

José-Manuel Fernández-Real jmfreal@idibgi.org

1 Department of Medical Sciences, CIBERobn Pathophysiology of Obesity and Nutrition, University Hospital of Girona, University of Girona, Girona, Spain

2 Tor Vergata Hospita, Rome, Italy

3 INSERM, Toulouse, France short chain fatty acids issued from the fermentation of dietary fiber, indols derivates from the metabolism of tryptophan and other aromatic aminoacids, or macromolecules such as peptidoglycans, LPS, or flagelins, recent evidences showed that intestinal bacteria do translocate to the adipose tissue to establish metabolic inflammation. Such observation suggest a leaky gut where bacteria would first stick to the intestinal mucosa of the small bowel and then translocate trancellularly or through the goblet cells to the lamina propria. The large population of innate and adaptive immune cells do consider the corresponding commensals as self, thereby not engaging a destruction program. The bacteria within the basal side of the intestine are phagocytosed by the innate immune cells that activate local adaptive interleukin-17 (IL17) producing $\mathrm{T}$ lymphocytes [9]. IL-17 is important for the production of defensins like Reg3 by epithelial cells that protect against large commensal invasions. On a daily basis the translocation of eubiotic gut microbiota avoids intense intestinal inflammation while still maintaining an affordable degree of intestinal defense. However, gut microbiota dysbiosis induce a deleterious bias where an impaired set of comensal bacteria translocate to the lamina propria. Through yet unknown mechanisms they reduce IL17 production avoiding the adaptative defense from the intestine to face the invasion by commensals. The latter, while still phagocytosed by the innate immune cells from the lamina propria do resist the destruction and remain intracellular. Upon a tissue stress such as a large fat accumulation (in adipose tissue or the liver or the arterial wall) the bacteria-loaded phagocytes do migrate to tissues where, while maintaining their proinflammatory capacity triggered by the indweled bacteria, they release cytokines, leading to metabolic inflammation, enhancing local and systemic tissue inflammation and fueling cardio-vasculometabolic diseases. Altogether, the physiological mechanism of bacterial translocation, that allows the maintenance of a vigilant state of the intestinal immune and epithelial defense, is overwhelmed by the gut microbiota dysbiosis and favors the triggering of metabolic 
inflammation within tissues. A tissue of interest in the control of cardiovascular and metabolic disease is the enteric nervous system and notably the gut brain axis. At the root of this gut brain axis, incretins, notably GLP-1, activate local enteric nervous system and the vagus nerve, informing the brain of a change in nutrient so that peripheral tissues get prepared for the managment of nutrients [10]. The liver, the muscle and adipose tissue will uptake glucose while the beta and alpha cells of the pancreas will regulate insulin and glucagon secretion for a fine tuning of the nutrient fluxes. Within this issue of REMD it is shown how a gut microbiota dysbiosis impairs enteric GLP-1-induced NO production by enteric neurons thereby affecting the activation of the gut-brain axis for the control of insulin secretion, gastric emptying and food intake. Germ free colonized mice were used to demonstrate the causality of the role played by gut microbiota. Along the same line of investigation, it has been described in humans that a low bacterial gene count in the gut was associated with liver steatosis in obese females. The gut microbiota dysbiosis led to the overt synthesis of aromatic amino acid derivates such as the phenylacetic acid, which triggered lipid accumulation in human hepatocytes [11]. The causal role of gut microbiota was shown by the transplantation of human microbiota from subjects with varying degree of hepatic steatosis, inducing the disease in rodents [11].

The gut microbiome-inflammation relationships may impact tissues as diverse as the oral mucosa [12], the heart [13] and the adipose tissue [14] and the enteric nervous system [15, 16] modifying their biology. Even the once considered sanctuary, the central nervous system, could be affected through the gut-brain axis [17], impacting on cognition in the context of direct and indirects mechanisms involving chronic inflammation [17].

The impact of oral microbiota dysbiosis is now regarded as a new concept with long term major impacts on cardiovascular and metabolic diseases. Periodontitis is associated with an oral microbiota dysbiosis that could hence be responsible for the triggering of metabolic inflammation. Chronic low grade infusion of LPS from the infected tooth generate a metabolic endotoxemia in the light of what has previously been observed with intestinal microbiota dysbiosis. Although the microbiota disversity of the oral cavity is much lower than that of the gut, the gram negative bacteria dominate, and their LPS was shown to be deleterious when chronically associated with a fat-enriched diet.

Beside the direct effect of gut and oral microbiota on cardiovascular disease, it is now appreciated that the microbiota produces numerous metabolites, some of which are absorbed into the systemic circulation and are biologically active, These metabolites trigger inflammatory pathways, thrombosis and cardiac arrhythmias, being new players in the complex process of atherosclerosis onset and progression [18].

Thus it seems that the microbiome should be taken into account in the equation when considering the pathophysiology of multiple metabolic diseases, that is increasingly recognized. In fact, a specific microbiota might have evolved in parallel with its host when struggling against changing environmental conditions [19].

\section{References}

1. Fernández-Real JM, Ricart W. Insulin resistance and inflammation in an evolutionary perspective: the contribution of cytokine genotype/phenotype to thriftiness. Diabetologia. 1999;42(11): 1367-74.

2. Fernández-Real JM, Ricart W. Insulin resistance and chronic cardiovascular inflammatory syndrome. Endocr Rev. 2003;24(3):278301.

3. Esteve E, Ricart W, Fernández-Real JM. Dyslipidemia and inflammation: an evolutionary conserved mechanism. Clin Nutr. 2005 Feb;24(1):16-31.

4. Stöhr R, Federici M. Insulin resistance and atherosclerosis: convergence between metabolic pathways and inflammatory nodes. Biochem J. 2013;454(1):1-11.

5. Cani PD, Amar J, Iglesias MA, Poggi M, Knauf C, Bastelica D, et al. Metabolic endotoxemia initiates obesity and insulin resistance. Diabetes. 2007;56(7):1761-72.

6. Cabrera-Mulero A, Tinahones A, Bandera B, Moreno-Indias I, Macías-González M, Tinahones FJ. Keto microbiota: a powerful contributor to host disease recovery. Rev Endocr Metab Disord. 2019. https://doi.org/10.1007/s11154-019-09518-8.

7. Schoeler M, Caesar R. Dietary lipids, gut microbiota and lipid metabolism. Rev Endocr Metab Disord. 2019. https://doi.org/10.1007/ s11154-019-09512-0 Review.

8. Fernández-Veledo S, Vendrell J. Gut microbiota-derived succinate: friend or foe in human metabolic diseases? Rev Endocr Metab Disord. 2019 Oct 25. https://doi.org/10.1007/s11154-019-09513-z.

9. Garidou L, et al. The gut microbiota regulates intestinal CD4 T cells expressing RORgammat and controls metabolic disease. Cell Metab. 2015;22:100-12.

10. Burcelin R. The gut-brain axis: a major glucoregulatory player. Diabetes Metab. 2010;36(Suppl 3):S54-8.

11. Hoyles L, et al. Molecular phenomics and metagenomics of hepatic steatosis in non-diabetic obese women. Nat Med. 2018;24:1070 80 .

12. Minty M, Canceil T, Serino M, Burcelin R, Tercé F, Blasco-Baque $\mathrm{V}$. Oral microbiota-induced periodontitis: a new risk factor of metabolic diseases. Rev Endocr Metab Disord. 2019 Nov 18. https:// doi.org/10.1007/s11154-019-09526-8.

13. Branchereau M, Burcelin R, Heymes C. The gut microbiome and heart failure: a better gut for a better heart. Rev Endocr Metab Disord. 2019. https://doi.org/10.1007/s11154-019-09519-7.

14. Moreno-Navarrete JM, Fernandez-Real JM. The gut microbiota modulates both browning of white adipose tissue and the activity of brown adipose tissue. Rev Endocr Metab Disord. 2019 Nov 27. https://oi.org/10.1007/s11154-019-09523-x. 
15. Grasset E, Burcelin R. The gut microbiota to the brain axis in the metabolic control. Rev Endocr Metab Disord. 2019 Oct 28. https:// doi.org/10.1007/s11154-019-09511-1.

16. Grasset E, et al. A specific gut microbiota dysbiosis of Type 2 diabetic mice induces GLP-1 resistance through an enteric NOdependent and gut-brain axis mechanism. Cell Metab. 2017;25: 1075-1090 e1075.

17. Arnoriaga M, Fernandez-Real JM. Microbiota impacts on chronic inflammation and metabolic syndrome - related cognitive dysfunction. Rev Endocr Metab Disord. 2019.
18. Kappel BA, Federici M. Gut microbiome and cardiometabolic risk. Reviews in Endocrine and Metabolic Disorders. In press. https:// doi.org/10.1007/s11154-019-09533-9.

19. Esteve E, Ricart W, Fernández-Real JM. Gut microbiota interactions with obesity, insulin resistance and type 2 diabetes: did gut microbiote co-evolve with insulin resistance? Curr Opin Clin Nutr Metab Care. 2011;14(5):483-90.

Publisher's note Springer Nature remains neutral with regard to jurisdictional claims in published maps and institutional affiliations. 